\title{
Breast Cancer by AJCC v7 Stage
}

National Cancer Institute

\section{Source}

National Cancer Institute. Breast Cancer by A/CC v7 Stage. NCI Thesaurus. Code C91230.

A term that refers to the staging of breast cancer according to the American Joint Committee on Cancer, 7 th edition. 